\title{
A NOTE ON THE KLEINECKE-SHIROKOV THEOREM AND THE WINTNER-WIELANDT-HALMOS THEOREM
}

\author{
C.-S. LIN
}

ABstract. We extend the Kleinecke-Shirokov theorem to the almost commutative case. From this result we prove the WintnerWielandt-Halmos theorem.

The Kleinecke-Shirokov theorem says, according to [1, p. 128], that if $P$ and $Q$ are bounded linear operators on a Hilbert space, $T=P Q$ $-Q P$ and $T$ commutes with $P$, then $T$ is quasinilpotent.

THEOREM 1. If $P$ and $Q$ are bounded linear operators on a Banach space $X, T=P Q-Q P$ and $T$ almost commutes with $P$, i.e., $P T-T P$ is a compact operator, then $T$ is a Riesz operator. In particular, if $X$ is a Hilbert space, then there exists a compact normal operator $S$ such that $T-S$ is a quasinilpotent operator.

Proof. We can prove the theorem by an analogous method to Kleinecke's original one $[1$, p. 335]. Let $c$ be the canonical homomorphism of $B(X)$, the Banach algebra of bounded linear operators on $X$, onto $B(X) / K(X)$, the quotient Banach algebra of $B(X)$ modulo $K(X)$, the closed two-sided ideal of compact operators on $X$. Let $c(P)$ be fixed and $D(c(Q))=c(P) c(Q)-c(Q) c(P)$ be a function of $c(Q)$, then $D$ is a bounded linear operator on $B(X) / K(X)$ which is also a derivation. By using the Leibniz formula and the fact that $D^{2}(c(Q))$ $=D(c(T))=c(P) c(T)-c(T) c(P)=0$, it can be shown that $D^{n}\left(c(Q)^{n}\right)$ $=n !(D(c(Q)))^{n}=n ! c(T)^{n}$. Hence $\left\|c(T)^{n}\right\|^{1 / n} \rightarrow 0$ as $n \rightarrow \infty$, i.e., $T$ is a Riesz operator [2]. The particular case will follow from the following proposition: If we denote by $R(X)$ the set of Riesz operators, $N(X)$ the set of quasinilpotent operators and $K M(X)$ the set of compact normal operators on $X$, then

$$
R(X)=N(X)+K M(X)=N(X)+K(X)=R(X)+K(X) .
$$

In fact, every Riesz operator on a Hilbert space is decomposable as the sum of a quasinilpotent operator and a compact normal operator $[3$, Theorem 7.5]. Hence $R(X) \subseteq N(X)+K M(X) \subseteq N(X)+K(X)$ $\subseteq R(X)+K(X)$. On the other hand, if $T \in R(X)$ and $S \in K(X)$, then

Received by the editors April 30, 1970.

AMS 1970 subject classifications. Primary 47B47; Secondary 47B05.

Key words and phrases. Almost commutative operators, Riesz operator, bounded derivation, Leibniz formula, commutator.

Copyright (c) 1971, American Mathematical Society 
$c(T+S)=c(T)$ is quasinilpotent, $T+S \in R(X)$ and hence $R(X)$ $+K(X) \subseteq R(X)$.

Theorem 2 (WintNer-Wielandt-Halmos TheOREM). If $b$ is $a$ nonzero scalar and $S$ is a compact operator on a Banach space $X$, then $b+S$ is not a commutator.

Proof. Suppose on the contrary that $b+S=P Q-Q P$ with $P$ and $Q \in B(X)$, then $P(b+S)-(b+S) P=P S-S P \in K(X)$. Hence $b+S \in$ $R(X)$ by Theorem 1 . But $-S \in R(X)$ and $(-S)(b+S)=(b+S)(-S)$, thus $b=(b+S)-S \in R(X)$ [2, Theorem 3.1]. By the definition of a Riesz operator, this is impossible unless $b=0$. This shows that $b+S$ is not a commutator.

If $S$ is the zero operator, Theorem 2 is precisely the WintnerWielandt theorem, i.e., the only scalar commutator is 0 .

\section{REFERENCES}

1. P. R. Halmos, A Hilbert space problem book, Van Nostrand, Princeton, N. J., 1967. MR 34 \#8178.

2. T. T. West, Riesz operators in Banach spaces, Proc. London Math. Soc. (3) 16 (1966), 131-141. MR 33 \#1742.

3. - The decomposition of Riesz operators, Proc. London Math. Soc. (3) 16 (1966), 737-752. MR 33 \#6417.

Queen's University, Kingston, Ontario, Canada 